\title{
Treatment for Patients with Severe Heart Failure after Coronary Artery Bypass Grafting Based on the Application Markov Model
}

\author{
X. CAO* \\ Tangshan Workers' Hospital, Tangshan, Hebei Province 063000, China
}

\author{
Cao et al.: Treatment for Severe Heart Failure after Coronary Artery Bypass Grafting Based \\ on Markov Model
}

\begin{abstract}
The Markov model is used to study the effect of the vascular geometric model and the elastic blood vessel wall on the treatment and nursing care for patients with severe heart failure after coronary artery bypass grafting due to changes in hemodynamic characteristics. In accordance with the original CT data of the patients undergoing the coronary artery bypass grafting, the data are imported to the MMICS software to construct the Markov model for application after the coronary artery bypass grafting. Markov model is applied on the basis of the two modules, the ANSYS mechanical module and the CFX module. Through the special fluid structure coupling algorithm, the bidirectional coupling calculation of elastic blood vessel wall and blood can be implemented. Under the condition of a given pulse pressure, the distribution and deformation status of the wall shearing stress of the elastic tube and the rigid wall are simulated and compared. The results of the research showed that the maximum wall shearing stress and the displacement of the Markov model are higher than those of the CAD simplified model. In addition, the positions where the maximum displacement occurs in the two models are completely different. The maximum wall shearing stress and displacement in the application Markov model are lower than those in the application of the rigid vessel wall model. These results could provide a more reasonable data reference for the clinical diagnosis of coronary restenosis
\end{abstract}

Key words: Markov model, severe heart failure, treatment and nursing care, coronary artery bypass grafting

With the improvement of people's living standards, the incidence of coronary atherosclerotic cardiopathy is gradually increasing, and coronary heart disease has seriously affects the daily activities and quality of life in the patients. The main method in cardiac surgery for the treatment of coronary atherosclerotic cardiopathy is the coronary artery bypass grafting. Coronary artery bypass grafting has been in existence for more than half a century and various surgical methods have been developed so far, such as the conventional extracorporeal circulation coronary artery bypass grafting, the mini extracorporeal circulation coronary artery bypass grafting, the off-pump coronary artery bypass grafting, the minimally invasive direct vision coronary artery bypass grafting, the robotic coronary artery bypass grafting and so on ${ }^{[1]}$. The incidence of restenosis in the arterial bridge within $10 \mathrm{y}$ is $5-10 \%$; and the incidence of occlusion in the venous bridge is even more common.
In the first year, nearly 15 to $30 \%$ of patients have restenosis and the incidence of obstruction is as high as $50 \%$ in $10 \mathrm{y}^{[2,3]}$. The coronary artery bypass grafting is closely related to the hemodynamic characteristics that lead to the endometrial hyperplasia ${ }^{[4]}$. For the conventional extracorporeal circulation coronary artery bypass grafting, in 1953, the United States successfully applied the extracorporeal circulation equipment independently developed to repair the atrial septal defect in the patients, which was the first time that the extracorporeal circulation equipment was used during the operation. It has opened the era for the surgeons to approach and repair the heart ${ }^{[5,6]}$. Coronary artery bypass grafting was performed for the first time using the extracorporeal circulation equipment in 1965. At present, the conventional extracorporeal coronary artery bypass grafting is still a technology that has been widely applied and generally accepted by the patients ${ }^{[7,8]}$. It has 
become a safe and mature technology. The conventional extracorporeal circulation device include 5 parts, the blood pump, the oxygenator, the temperature changer, the blood storage chamber and the filter. The blood is discharged from the vein into a large-volume storage chamber where the blood is mixed with air, and oxygen is supplied through the membrane oxygenator and the appropriate pressure is supplied by a roller pump to pump the blood back to the aorta ${ }^{[9,10]}$. The conventional extracorporeal circulation stops the heart from beating, which can facilitate the surgeon to perform fine coronary artery bypass between the stenotic artery and a patent coronary artery. The vascular patency rate after the coronary artery bypass grafting using the convention extracorporeal circulation is acceptable. In the case where the left iliac artery is used for the extracorporeal coronary artery bypass grafting, the 1-year patency rate is more than $90 \%$. In case where the internal mammary artery is used for the on-pump coronary artery bypass grafting, the 10-year patency rate is more than $80 \%$ and the clinical symptoms of myocardial ischemia in these patients have been alleviated to a great extent. The inadequacy of the conventional extracorporeal circulation is that the pre-filling requires a large amount of crystalloid and colloidal fluid, which is highly likely to lead to postoperative low hemoglobin concentration and low hematocrit. In the extracorporeal circulation, the formation of blood is disrupted, denaturation of the plasma protein occur and the blood is in contact with large areas of tubing, containers and air to trigger the complement activation. This will alter the cytokine stability state and the coagulation and fibrinolysis systems, activate the immune-active cells and cause the vascular endothelial injury. Hence, the establishment of an accurate coronary artery bypass grafting model is the key to the hemodynamic studies ${ }^{[1,12]}$. However, the real coronary vessels are elastic and the fluid pressure can cause the deformation of the blood vessels. The variation in the diameter of the blood vessels can be up to $10 \%$ in the order of magnitude, which can even reach more than $20 \%$ in the aorta. On the other hand, the deformation of the blood vessels in turn will affect the flow of blood ${ }^{[13,14]}$. The treatment and nursing care for patients with severe heart failure is an important factor that affects the hemodynamic characteristics and requires in-depth research ${ }^{[15,16]}$. The research on the numerical simulation based on the application Markov model can provide a more reasonable data reference for the clinical diagnosis of the coronary restenosis ${ }^{[17]}$. In this paper, on the basis of the CT image data of the patients, a 3D application Markov model after the coronary artery bypass grafting is reconstructed. In addition, the 3D numerical simulation is carried out by using ANSYS software and the CFX software to compare and analyze the effects of the geometric model and the treatment and nursing care for patients with severe heart failure on the hemodynamic characteristics.

\section{MATERIALS AND METHODS}

The subject selected is a patient undergoing the coronary artery bypass grafting in Beijing Fuwai Cardiovascular Hospital, who is a female, 56 y old, with left coronary artery occlusion, underwent the transplantation of great saphenous vein. Fifteen days after the bypass grafting, an application Markov model is constructed. The 64-slice multi detector spiral CT scanner by GE Co., USA was used to carry out fault segmentation on the human chest (slice thickness is $0.5 \mathrm{~mm}$, a total of 466 scans) and the medical images in the DICOM format were obtained. The CT coronary angiography is shown in fig. 1.

\section{3-D reconstruction of application Markov model after coronary artery bypass grafting:}

The CT scan data of the patients who underwent the coronary artery bypass grafting was imported into the MIMICS software and the images are positioned automatically. The approach of combining the regional growth method based on the gray value, the threshold segmentation method and the manual segmentation method was adopted and then optimization treatment was carried out on the number of the surfaces and the quality of the surface model to obtain the application Markov model optimized after the coronary artery bypass grafting. The thickness of the CT image acquisition layer is relatively high and the gray value of the coronary artery blood vessels was relatively close to that of the other tissues and entangled on the heart. Therefore, the extraction process of the model is relatively time-consuming and laborious. In order to carry out the fluid structure coupling calculation in the ANSYS software and the CFX software, it is necessary to have the vessel wall surface model in the format of NURBS. For the CT images obtained by the tomography scanning, it is impossible to identify the wall thickness of the blood vessel. It is required that the STL should be relatively thin and irregular. Hence, it is very easy to cross when constructing the grid and it takes relatively a long time to complete the repair processing manually. The application Markov model thus generated is shown in fig. 2. It can be imported into the ANSYS Geometry to generate a blood flow 
model by using its Fill function, which has the degree of stenosis of about $75 \%$. In accordance to the trend of vascular spline curve after the coronary artery bypass grafting, the simplified CAD model with the blood vessel diameter of $2 \mathrm{~mm}$ is plotted by using the UG 3-D graphics software, and the diameter of the vessel at the occlusion site is $1 \mathrm{~mm}$. Shelling is carried out in the ANSYS Geometry with the wall thickness of $0.1 \mathrm{~mm}$. The resulting wall model after the coronary artery bypass grafting thus generated is shown in fig. 3 . Then, the Fill function is used to fill the inner surface of the vessel wall to generate a blood flow model.

\section{Treatment and nursing care for patients with severe heart failure after coronary artery bypass grafting:}

The 3-D software was used to reconstruct the application Markov model, which cannot separate 2 kinds of blood vessel walls and thus different material properties are assigned. Although the wall properties of the great saphenous vein and the coronary artery indicate that both have elastic walls, relative to a rigid wall, the difference in the parameters of the 2 kinds of elastic walls have no substantial influence on the study. Therefore, uniform material property data was adopted, which was set as the following, the density of the vessel wall is $1,062 \mathrm{~kg} / \mathrm{m}^{3}$, the elastic modulus is $4.66 \mathrm{MPa}$, and the Poisson's ratio is 0.45 , and the density of blood is $1,050 \mathrm{~kg} / \mathrm{m}^{3}$. The blood under physiological condition is a kind of viscoelastic suspension medium. It is a multi-phase system that is composed blood cells and the plasma. The viscosity of the blood is dependent on the concentration of the red blood cells, their deformability, aggregation, viscosity of the plasma and so on and it falls under the category of a non-Newtonian fluid. In the hemodynamic studies at present, blood is generally assumed to be a viscous Newtonian fluid. This assumption is appropriate for the case of the high flow shearing rate. However, in case of the presence of stenosis in the small blood vessels or blood vessels, the region that generates very low flow shearing rate may exist. Hence, it is necessary to take the non-Newtonian properties of the blood into consideration. Researchers have put forward a few stress-strain relationship models related to the viscosity of the blood. However, no single model can describe the true rheological characteristics of the blood accurately. The Carreau non-Newtonian model is considered to be very close to the experimental blood flow environment. In addition, its non-Newtonian effect is not over estimated. Therefore, the Carreau model is adopted in this paper to express the non-Newtonian properties of the blood, and the viscosity expression is as follows, $\eta(\gamma \&)=\eta_{\infty}+\left(\eta_{0}-\eta_{\infty}\right)\left[1+(\lambda \gamma \&)^{\mathrm{a}}\right]^{(\mathrm{n}-1 / \mathrm{a})}$. In this Eqn., time constant $\lambda=3.313 \mathrm{~s}$; zero shear viscosity $\eta_{0}=0.056$ $\mathrm{Pa} \cdot \mathrm{s}$; infinite shear viscosity $\eta_{\infty}=0.00345 \mathrm{~Pa} \cdot \mathrm{s}$; power exponent $\mathrm{n}=0.3568$; and constant $=2$.

\section{Governing equation:}

Blood is regarded as an unsteady, non-Newtonian, incompressible laminar fluid, then the momentum Eqn. and the Navier-Stokes equation are as the following respectively $\nabla_{\mathrm{gv}}=0 \rho_{\mathrm{f}}\left(\partial_{\mathrm{v}} / \partial_{\mathrm{t}}+\left(\mathrm{v}-\mathrm{v}_{\mathrm{g}}\right) \mathrm{g} \nabla_{\mathrm{v}}\right)=-\nabla_{\mathrm{p}}+\nabla_{\mathrm{gT}}$, where $\nabla$ stands for the Hamilton operator; v stands for the fluid velocity vector; $v_{g}$ stands for the moving grid speed; $\mathrm{T}$ stands for the stress tensor; $\mathrm{p}$ stands for the fluid pressure and $p_{f}$ stands for the fluid density, where the effect of the physical strength is not taken into consideration. In the fluid structure coupling, the governing Eqn for the motion of the vessel wall is as the following $\nabla \cdot \sigma_{s}=\rho_{s} a_{s}$, where $\sigma_{s}$ stands for the stress tensor of the vessel wall; $p_{s}$ stands for the density of the vessel wall and $a_{\mathrm{s}}$ stands for the acceleration of the vessel wall. The fluid pressure, displacement and so on are transmitted between the blood and the vessel wall through the interface. Therefore, the following conditions should be met at the interface: $d_{s=} d_{f \text { and }}$ $\sigma_{s} g n_{s}=\sigma_{f} g n_{f}$ In the Eqn. $\mathrm{d}$ stands for the displacement vector; $\sigma$ stands for the stress tensor; $\mathrm{n}$ stands for the normal direction of the boundary; the subscripts $f$ and $s$ stand for the fluid and the solid, respectively.

\section{Boundary conditions:}

As the data of the patient does not include the blood flow of the coronary artery and the bypass graft determined by the instant blood flow meter, average pulsating velocity of the inlet section is adopted for both the coronary artery and the graft vessel. The curve of the change in the inlet velocity with time is shown in fig. 4. The cardiac cycle is $0.8 \mathrm{~s}$. In accordance with the condition for the sufficient flow development, the pressure at the coronary artery outlet is set to $0 \mathrm{~Pa}$. The Reynolds number of the blood flow in the coronary artery is $\mathrm{R}_{\mathrm{e}}=\rho_{\mathrm{f}} \mathrm{v}_{\mathrm{i}, \max } \mathrm{D} / \eta \approx 200$, in which $v_{i, \max }$ and $\mathrm{D}$ stand for the maximum velocity at the inlet of the coronary artery and the diameter of the blood vessel, respectively. $R_{e}<2300$, the state of the blood flow in the coronary artery is laminar flow. The slip-free boundary condition is adopted for the blood and the blood vessel wall. The inlet and outlet of the coronary artery and the inlet of the graft vessel are connected to the upstream and the downstream vessels, respectively, in which the fully fixed constraint is adopted. In order to improve 
the accuracy of the calculation after the coronary artery bypass grafting, two cycles of the iterative calculation are carried out continuously, and the results in the latter cardiac cycle are selected for analysis as the following.

\section{RESULTS AND DISCUSSION}

Endothelial cell proliferation in the failure of the coronary artery bypass grafting is closely related to the local hemodynamic factors; while the intravascular local hemodynamics is strongly dependent on the geometry of the coronary artery bypass graft vessel. The increase in the blood flow in the graft is a gradual process. Part of the blood flow passes through the stenotic coronary artery in the early stage after the coronary artery bypass grafting. The confined jet that comes from the stenosis interacts with the blood flow that comes from the graft in the vicinity of the suture area and forms complicated flow field. This will have an important effect on the endothelial cell proliferation in the suture area and the re-occlusion at the site of stenosis. Due to sudden changes in the condition, patients often have negative emotions such as nervousness, anxiety, fear, despair and so on. These negative emotions will increase the oxygen consumption of the myocardium and thus affect the treatment of the disease. Therefore, nurses should have keen observation and carry out counselling in a timely manner to dispel the negative emotions of the patients. During the rescue process, nurses should make appropriate explanations and provide sincere care that include, giving the patients a vote of belief, wiping the sweat from the forehead of the patients, holding both hands of the patients tightly, carrying out professional and skilled nursing operations and so on, so as to gain the trust of the patients, help them build confidence in fighting against the disease and facilitate active cooperation of the patients with the treatment. The fact that the motion of the vessel wall can reflect the blood flow in the blood vessel more truthfully is taken into consideration. For the application Markov model, the individualized rigid vessel wall model, the CAD simplified model and the CAD simplified nonapplication Markov model, the effects of the different model conditions on the wall shearing stress and the wall displacement are studied.

The wall shearing stress plays a crucial role in the formation and development of the endometrial hyperplasia. Fig. 5 shows the distribution cloud chart of the wall shearing stress after the coronary artery bypass grafting under different models at the time when the inlet velocity is the peak value $(t=0.15 \mathrm{~s})$. The models include the application Markov model, the individualized rigid vessel wall model, the CAD simplified model and the CAD simplified rigid vessel wall model. In general, the variation trend of the wall shearing stress distribution of the four models is basically the same, but the wall shearing stress gradient is relatively large. In particular, at the coronary artery occlusion site, the maximum value of the wall shearing stress is present and it is much higher than that in the normal state of the human body. Studies have shown that greater wall shearing stress will lead to more probability to cause the endometrial hyperplasia, which will further cause the vascular restenosis. The occlusion at the coronary artery stenosis will aggravate the reduction in the blood flow. Hence the blood flow in the vascular graft will increase relatively, and the longterm patency rate will increase accordingly.

Fig. 6 shows the change relationship of the maximum value of the wall shearing stress at the time when the outlet velocity is the peak velocity $(t=0.15 \mathrm{~s})$ with the coronary artery bypass vessel model. It can be seen that the maximum value of the wall shearing stress in the application Markov model at the occlusion position is higher than the results of the CAD simplified model. The reason is that the application Markov model is caused by the irregular rounded tubes with local protrusions and depressions. The elastic vessel wall motion has weakened the shearing force of the fluid on the vessel wall, which results in the fact that the maximum value of the wall shearing stress in the elastic vessel wall model is lower than that in the rigid vessel wall model. Hence, it can be seen that when the CAD simplified model based on the rigid vessel wall which is simplified directly after the coronary artery bypass grafting is compared with the individualized elastic vessel wall model, there is relatively great deviation in the distribution situation of the wall shearing stress as the following.

Fig. 7 is the cloud chart of the displacement of the wall after the coronary artery bypass grafting at the time when the inlet velocity during the cardiac cycle selected is the peak value $(t=0.15 \mathrm{~s})$ with the maximum displacement of the vessel wall (that is, the site where the vascular rupture is most prone to occur). The irregular shape of the blood vessel has caused the maximum displacement value of the wall in the application Markov model to be $3.323 \times 10^{-5}$, which is higher than the $3.045 \times 10^{\text {- }}$ 5 in the CAD simplified model, close to $8.4 \%$. This indicated that the vascular intima can be injured at the maximum displacement site of the vessel wall in the application of the CAD simplified model (that is, the 
site where vascular rupture is most prone to occur). At this point, degeneration, necrosis and exfoliation of the endothelial cells could occur and the collagen fibers under the endothelium would be exposed. In addition, the damaged endometrium would become rough, which could make it easier for the platelets to adhere and form thrombus to block the blood vessel. The positions of the maximum displacement of the wall are also very different to generate. The maximum displacement in the application Markov model was present in the graft vessel and the suture area. In addition, the range of the rupture would be relatively small, which was consistent with the position and condition of the coronary artery restenosis observed clinically in practice. However, the maximum displacement value of the application Markov model is at the lateral curvature of the coronary artery and the graft vessel. The reason is that the velocity of the blood flow at the lateral curvature vessel is higher than the velocity of the blood flow in the medial vessel. Therefore, it can be seen that the results calculated by the application Markov model have large deviation in the maximum displacement value of the wall obtained by the real vascular model as well as the position where the maximum displacement occurs from the real situation. Hence, the application Markov model with more practical value shall be adopted in the specific clinical practice guidance.

Heart failure is a kind of emergency with sharp decline in myocardial contractility caused by a variety of reasons, which results in a sudden drop in the cardiac output and the failure to meet the needs of the body, leading to congestion in the venous system and insufficiency of blood supply to the arterial system. The symptoms of the disease are serious and the condition is dangerous. Without rescue in a timely manner, the patient's life is often endangered. In view of the treatment and nursing care for patients with severe heart failure after coronary artery bypass grafting, through the construction and application Markov model method, studies are carried out on the effects of the vascular geometric model and the elastic blood vessel wall on the treatment and nursing care for the patients with severe heart failure after the coronary artery bypass grafting due to the change in the hemodynamic characteristics. Regardless of the elastic wall surface or the rigid wall surface, the maximum wall shearing stress in the CAD simplified model is higher than the results in the application Markov model. In the two kinds of vascular geometric models, the maximum value of the wall shearing stress in the elastic vessel wall model is lower than the results in the rigid vessel wall model. The maximum wall displacement of the Markov model is higher than that in the CAD simplified model. The positions of the maximum wall displacement generated in the two kinds of the vascular geometric models also have very significant difference. The maximum displacement in the application Markov model is present at the bypass graft and the suture area and the range is relatively small; while the maximum displacement in the CAD simplified model is at the bend of the blood vessels. The application Markov model after the individualized coronary artery bypass grafting can reflect the hemodynamic characteristics more truthfully than the CAD simplified model of the relatively rigid wall, which has provided the clinical surgeons with an auxiliary means for the diagnosis of the pathological degree and the site of restenosis after the coronary artery bypass grafting.

\section{REFERENCES}

1. Boudoulas H, Lewis RP, Snyder GL, Karayannacos P, Vasko JS. Beneficial effect of continuation of propranolol through coronary bypass grafting. Clini Cardiol 2015;2(2): 87-91.

2. Kamalesh M, Sharp TG, Tang XC, Shunk K, Ward HB, Walsh $\mathrm{J}$, et al. Percutaneous coronary intervention versus coronary bypass grafting in united states veterans with diabetes. J Am Coll Cardiol 2013;61(8):808-16.

3. Hlatky MA, Solomon MD, Shilane D, Leong TK, Brindis $\mathrm{R}$, Go AS. Use of medications for secondary prevention after coronary bypass grafting compared with percutaneous coronary intervention. J Am Coll Cardiol 2013;61(3):295-301.

4. Seifalian A, Tiwari A, Hamilton G, Salacinski H. Improving the clinical patency of prosthetic vascular and coronary bypass grafts: the role of seeding and tissue engineering. Artif Organs 2015;26(4):307-20.

5. Hamada Y, Kawachi K, Nakata T, Kohtani T, Takano S, Tsunooka N. Antiinflammatory effect of heparin-coated circuits with leukocyte-depleting filters in coronary bypass grafting. Artif Organs 2015;25(12):1004-8.

6. Itagaki S, Cavallaro P, Adams DH, Chikwe J. Bilateral internal mammary artery grafts, mortality and morbidity: an analysis of 1526360 coronary bypass operations. Heart 2013;99(12):84953.

7. Sastry P, Rivinius R, Harvey R, Parker RA, Rahm AK, Thomas $\mathrm{D}$, et al. The influence of endoscopic vein harvesting on outcomes after coronary bypass grafting: a meta-analysis of 267525 patients. Eur J Cardiothorac Surg 2013;44(6):980-9.

8. Jr FT, Chen C, Buch AN. Fractional flow reserve-guided coronary bypass grafting: should surgeons use it? Curr Opin Cardiol 2013;28(6):654-60.

9. Li Y, Cai X, Mukamel DB, Cram P. Impact of length of stay after coronary bypass grafting on short-term readmission rate: an instrumental variable analysis. Med Care 2013;51(1):45-51.

10. Engoren M, Schwann TA, Habib RH, Neill SN, Vance JL, Likosky DS. The independent effects of anemia and transfusion on mortality after coronary artery bypass. Anna Thorac Surg 2014;97(2):514-20.

11. Gheorghiade M, Vaduganathan M, Fonarow, GC, Bonow, RO. Rehospitalization for heart failure. J Am Coll Cardiol 2013;61(4):391-403.

12. Anna J, Emma PL, Bengt, F. Malnutrition in patients suffering 
from chronic heart failure; the nurse's care. European Journal of Heart Failure 2001;3(4):449-56.

13. Akao H, Polisecki E, Kajinami K, Trompet S, Ford I, Jukema JW, et al. The abcal gene variant and heart disease risk reduction in the elderly during pravastatin treatment. Atherosclerosis 2014;235(1):176-81.

14. Alosco ML, Spitznagel MB, Van DM, Raz N, Cohen R, Sweet LH, et al. Cognitive function and treatment adherence in older adults with heart failure. Psychosom Med 2012;74(9):965-73.

15. Strömberg A, Wodlin P. Cardiopulse; pocket-sized ultrasound for nurses in heart failure? Eur Heart J 2016;37(5):434-5.

16. Ma R, Shi JS, Liu JC, Zhang YL. Establishment and application of cloud-markov model based on aquifer hydraulic conductivity. J Hydraul Eng 2012;43(7):767-76.
17. Yuksel SE, Gader PD. Context-based classification via mixture of hidden markov model experts with applications in landmine detection. IET COMPUT VIS 2017;10(8):873-883.

This is an open access article distributed under the terms of the Creative Commons Attribution-NonCommercial-ShareAlike 3.0 License, which allows others to remix, tweak, and build upon the work non-commercially, as long as the author is credited and the new creations are licensed under the identical terms

This article was originally published in a special issue: Special issue on "Drug Development and Human Health in China"

Indian J Pharm Sci 2020:82(1)spl issue4;19-25 